\section{The verb as the main determinant of sentence meaning*}

\author{
ALICE F. HEALY and GEORGE A. MILLER \\ The Rockefeller University, New York, N.Y. 10021
}

Ss sorted simple active sentences which differed only in their agents and verbs into piles on the basis of similarity of meaning. All the sentences that had the same verb but different agents were placed together into one pile by most Ss. It is concluded that the verb of a sentence influences the meaning of the sentence more than does the agent.

Certainly the meaning of a sentence is determined by the meanings of each word or morpheme in the sentence. Yet the meaning of the whole sentence is not just a linear summation of the meanings of each of its parts. Different parts of speech influence the meaning of a sentence differentially. More specifically, it is likely that the main verb of a sentence is more closely linked to the meaning of the whole sentence than is either the noun acting as subject or that acting as direct object.

The aim of the present experiment is to determine if the main verb of a sentence is more influential in determining the judged meaning of a sentence than is the noun used as agent. The present experiment employs the method of sorting similar to that described by G. A. Miller (1967). Simple active sentences which differed only in their subjects and verbs were sorted into piles on the basis of "similarity of meaning."

Pilot studies indicated that the choice of agents and verbs used in the experiment was critical. Ss often either sorted by agents, putting all the sentences with the same agent together into one pile, or sorted by verbs, putting all the sentences with the same verb into one pile. $S$ had been instructed to put into one pile the sentences he felt were most similar in meaning. Therefore S's grouping in to one pile sentences with different verbs but the same agent would indicate that he judges that verbs are less important in determining sentence meaning than are agents. Similarly his placing into one pile sentences with different agents but the same verb would indicate that he judges that agents do not contribute as much to sentence meaning as do verbs. The pilot research showed that Ss had a tendency to sort the sentences by agents if the agents chosen were farther apart in meaning than were the verbs and by verbs if the verbs were farther apart in meaning. For example, if the agents were everyone and his friend and the verbs were recalled and remembered, the sentences would be sorted by the agents. On the

*This investigation was supported in part by PHS Grant No.5T01 GM01789-04 BHS and ARPA Grant No. DAHC 15 to The Rockefeller University. other hand, if the agents were the book and the volume and the verbs were referred and fell, the sentences would be sorted by verbs. Therefore, the present experiment imposed the condition that the agents be as far apart in meaning as are the verbs. Further pilot studies showed that if Ss were not constrained as to the number of piles they were allowed to use, they would be less likely to sort either by agents or by verbs. A suitable constraint was thus imposed in the present experiment. Moreover, a tendency for Ss to sort sentences on the basis of "likelihood" or "plausibility" was observed in pilot work. The present experiment tries to overcome this problem without biasing the $\mathrm{Ss}$ in favor of either agents or verbs by requiring the Ss to separate the most plausible sentences.

\section{SUBJECTS}

Sixteen male and female young adults who had responded to advertisements in local newspapers served as $\mathrm{Ss}$ in this experiment.

\section{MATERIALS}

Twenty-five $3 \times 5$ in. white index cards each card was one English sentence. Each of the 25 sentences that appeared on the index cards was a simple active sentence with one of five subjects-the salesman, the critic, the writer, the student, the publisher-one of five verbs-sold, criticized, wrote, studied, published-and the book as direct object. The 25 sentences included all of the 25 possible combinations of the five subjects with the five verbs. The meaning of each of the five possible subjects corresponded to that of each of the five possible verbs to satisfy the condition that the distance in meaning between each pair of agents be equal approximately to the distance in meaning between each corresponding pair of verbs. For example, the salesman is assumed to be as distant in meaning from the critic as sold is to criticized.

Ss were tested individually. The following instructions were read by $E$ to each $S$ at the start of each experimental session: "Here are 25 cards with an English sentence on each card. I want you to sort were employed. Typed in the center of these cards into five piles on the basis of similarity of meaning so that the sentences that are closest together in meaning are in the same pile. In order to help you decide which cards to put into which piles, I will put one card into each of the five piles." $\mathrm{E}$ then placed into separate piles in front of $S$ the five cards with the following sentences on them: "The salesman sold the book." "The critic criticized the book." "The writer wrote the book." "The student studied the book." "The publisher published the book." These five sentences were chosen since they were felt to be the "most plausible" sentences. In addition, the agents and verbs chosen for each sentence were those which corresponded in meaning. Therefore, this selection of sentences did not bias the $\mathrm{S}$ to sort either by agents or by verbs.

After placing the five cards in front of $\mathrm{S}$, E shuffled the remaining 20 cards and handed them to $S$, who sorted the cards. $S$ was given as much time as he desired to finish sorting the cards into the five piles. RESULTS

Thirteen of the 16 Ss sorted the cards by verbs so that within each pile of the five piles the verbs in each sentence were identical. Two of the 16 Ss sorted the cards by agents so that within each of the five piles the subjects in each sentence were identical. One $\mathrm{S}$ sorted the cards into five piles using a mixed strategy so that within each pile there was a mixture of both verbs and agents. Yet in the process of sorting, this $\mathrm{S}$ rearranged the original five cards placed by $E$ so that two of them were in the same pile. Therefore, the results of this $\mathrm{S}$ were discarded. Using the results of the other $15 \mathrm{Ss}$, a sign test. is significant at the .008 level, enabling one to reject the null hypothesis of no difference between agents and verbs as a basis for card sorting.

\section{DISCUSSION}

The results of the present experiment point to a significant difference between subjects and verbs and their relation to the meaning of a sentence. Verbs appear to influence the judged meaning of a sentence to a greater extent than do nouns used as agents. Since active sentences were used exclusively, the agents were always at the start of each sentence. An explanation of the experimental findings in terms of the surface prominence of the verb's position in a sentence is therefore impossible. A more promising start toward explaining the results is in terms of the verb's function. To use a theatrical metaphor, the main verb of a sentence defines the plot; the subject merely indicates one of the actors REFERENCE

MIL LR, G. A. Psycholinguistic approaches to the study of communication. In D. L. Arm (Ed.), Journeys in science. Albuquerque: The University of New Mexico Press, 1967. Pp. 22-73. 